\title{
Wave Functions, Creation and Annihilation Operators of Quantum Physical System
}

\author{
Malkhaz Mumladze \\ Gori University, Gori, Georgia \\ Email: mmumladze@mail.ru
}

How to cite this paper: Mumladze, M. (2019) Wave Functions, Creation and Annihilation Operators of Quantum Physical System. Open Access Library Journal, 6: e5166.

https://doi.org/10.4236/oalib.1105166

Received: January 3, 2019

Accepted: January 19, 2019

Published: January 22, 2019

Copyright $\odot 2019$ by author(s) and Open Access Library Inc.

This work is licensed under the Creative Commons Attribution International License (CC BY 4.0).

http://creativecommons.org/licenses/by/4.0/

\begin{abstract}
In this article we proposed derivation of the wave function of a quantum system from the functional representation of the state of this system. There is considered representation of creation and annihilation operators by boundary and co-boundary operators of chain and co-chain complexes on the physical space.
\end{abstract}

\section{Subject Areas}

Modern Physics, Quantum Mechanics

\section{Keywords}

Quantum, Physical, System, State, Pure State, Observable, Measure, Projector, Chain Complex, Co-Chain Complex, Boundary and Co-Boundary Operators

\section{Introduction}

A quantum physical system can represented by a couple $(U, \mathfrak{I})$, where $U$ is some $C^{*}$-algebra which Hermitian elements that are called observables, and some subset $\mathfrak{I} \subset E_{U}$ of the set positive functionals on $U$ with norm one called the quantum states of this physical system [1] [2] [3].

Denote by $P_{U}$, the set of pure states on $C$-algebra $U, P_{U} \subset E_{U}$.

In the set of all linear continue functional on $C$-algebra $U$ we have topological structure which is called as ${ }^{\star}$ weakly topological structure [2]. Accordingly, in the set $P_{U}$ we have the topological structure induced from this.

Denote by $\Re$ the set of Hermit's elements of $U C^{\star}$-algebra.

Let $\left\{p_{\alpha}\right\}$ be the set of all one dimensional projectors on $C^{*}$-algebra $U$ and $\omega$ pure state, in the work [4] we shove that pure state has the meaning 1 only on one one-dimensional projector $p_{\alpha} \in\left\{p_{\alpha}\right\}$ and the meaning 0 on the other 
one dimensional projectors. Denote pure state which on projector $p_{\alpha}$ has the meaning 1 , so $\omega_{\alpha}$.

Every state $\omega \in \mathfrak{I}$ in space $P_{U}$ with weakly topological structure is defined on the Borel $\sigma$-algebra of a probability measure $\mu_{\omega}:\left\{\left\{p_{\alpha_{\beta}}\right\}\right\} \rightarrow R$ which is defined by the equality $\mu_{\omega}\left(\left\{p_{\alpha_{\beta}}\right\}\right)=\|v\|$ where $v$ positive functional $\|v\| \leq\|\omega\| \leq 1$, whose values on the elements of this subset $\left\{p_{\alpha_{\beta}}\right\}$ are coincident with corresponding values of the state $\omega$ [4].

For Hermit's elements $u \in \mathfrak{R}$ we have representation

$$
u=\int_{-\infty}^{\infty} \lambda \mathrm{d} p_{\lambda}^{u}
$$

where $\lambda$ is element of spectrum $\sigma_{u}$ and $p_{\lambda}^{u}$ is element of partition of unity of Hermit's element $u \in \mathfrak{R}$ [5]. It follows that for all $\omega \in E_{U}$ we have $\omega(u)=\int^{\infty} \lambda \mathrm{d} \omega\left(p_{\lambda}^{u}\right)$, and for the pure states $\omega_{\alpha} \in P_{U}$ we have placed the equality $\omega_{\alpha}\left(\bar{u}^{\alpha}\right)=\lambda_{\alpha}^{u}$, where $\lambda_{\alpha}^{u}$ is some element of spectrum of Hermit element $u \in \Re$. The last equality $\omega_{\alpha}(u)=\lambda_{\alpha}^{u}$ gives opportunity identify every pure state $\omega_{\alpha} \in P_{U}$ with the set of number $\left\{\lambda_{\alpha}^{u}\right\}_{u \in \Re}$.

The $\left\{\lambda_{\alpha}^{u}\right\}_{u \in \Re}$ indexed set is subset of The Tikhonov's product $\Sigma={\underset{u \in \Re}{\otimes}}_{u} \sigma_{u}$, where $\sigma_{u} \subset R$ spectrum of element $u \in \Re$. It fallows that $P_{U} \subset \Sigma=\underset{u \in \Re}{\otimes} \sigma_{u}$. So in the set $P_{U}$ we have topological structure induced from Tikhonov's product $\Sigma=\underset{u \in \mathcal{R}}{\otimes} \sigma_{u}$. This topological structure coincides with the induced topological structure from ${ }^{*}$ weakly topological structure on set of functionals on $C^{*}$ algebra $U$.

Let Support $\mu_{\omega}$ be the support of the measure $\mu_{\omega}$. If given physical quantum system $(U, \mathfrak{I})$, where each state $\omega \in \mathfrak{I}$ represents some elementary particle, this elementary particle corresponds to set of pure states, Support $\omega$. This means that elementary particles of system $(U, \mathfrak{I})$ are located in subspace $P_{\mathfrak{I}} \subset P_{U}$, where

$$
P_{\mathfrak{I}}=\bigcup_{\omega \in \mathfrak{I}} \text { Support } \omega,
$$

let us call the subspace $P_{\mathfrak{I}}$ by physical space of the physical quantum system $(U, \mathfrak{I})$.

In future physical quantum system we represent as triple $\left(U, P_{\mathfrak{I}}, \mathfrak{I}\right)$.

\section{Wave Function, Interference of Identical Elementary Particles}

Let given quantum physical system $\left(U, P_{\mathfrak{I}}, \mathfrak{I}\right)$.

Remark. We defined the wave function when the physical space $P_{\mathfrak{I}}$ coincides with $P_{U}, P_{\mathfrak{I}}=P_{U}$, we can also similarly define it then when the physical space is $P_{\mathfrak{I}} \subset P_{U}$, because $\mu_{\omega}\left(P_{\mathfrak{I}}\right)=1$ if $\omega \in \mathfrak{I}$.

This set of one dimensional projectors in $U$ we can identify to the set of pure states $P_{U}$. Thus, $\{p\} \approx P_{U}$. It follows, that for every state $\omega \in \mathfrak{J}$ exist the function 


$$
\begin{aligned}
& \varphi_{\omega}: P_{U} \rightarrow R, \varphi_{\omega}\left(\omega_{p}\right)=\omega(p) \geq 0, \\
& \omega_{p}\left(\omega_{p^{\prime}}\right)=0 \text { if } p \neq p^{\prime}, \omega_{p}\left(\omega_{p^{\prime}}\right)=\omega_{p}(p) \text { if } p=p^{\prime}
\end{aligned}
$$

where $\omega_{p}$ the pure state which corresponds to one dimensional projector $p$. Further, where does not cause confusion for to indicate the pure state $\omega_{p}$ or one-dimensional projector $p$ we will use the symbol $p$. As known if $\omega \in E_{U}$ state then $\omega(I)=1$ where $I$ is identical operator.

We have $\omega(u)=\int_{-\infty}^{\infty} \lambda \mathrm{d} \omega\left(p_{\lambda}^{u}\right)=\int_{-\infty}^{\infty} \lambda \omega\left(p_{\lambda}^{u}\right) \mathrm{d} \lambda$. Functional $\omega \in \mathfrak{I}$ is state, therefore, $\int_{-\infty}^{\infty} \omega\left(p_{\lambda}^{u}\right) \mathrm{d} \lambda=1$. Consider the measure $\mu_{\omega}$ on $P_{U} \subset \Sigma=\otimes_{u \in \Re}^{\otimes} \sigma_{u}$, $\omega(\tilde{p})=\int_{\bar{p}} \omega(p) \mathrm{d} \mu_{\omega}$ where $\bar{p}$ is set of such one dimensional projectors $\bar{p}=\{p\}$ that make up projector $\tilde{p}, \quad \tilde{p}=\bigoplus_{p \in \bar{p}} p$. It follows $\omega(u)=\int_{-\infty}^{\infty} \lambda \mathrm{d} \omega\left(p_{\lambda}^{u}\right)=\int_{-\infty}^{\infty} \lambda\left(\int_{\bar{p}_{\lambda}^{u}} \omega(p)\right) \mu_{\omega} \mathrm{d} \lambda$, where $\bar{p}_{\lambda}^{u}$ is set of such one dimensional projectors $\bar{p}=\{p\}$ that make up projector $p_{\lambda}^{u}=\underset{p \in \bar{p}}{\oplus} p$. Everything saying there, follows that $\omega=\int_{P_{U}} p \varphi_{\omega}(p) \mathrm{d} \mu_{\omega}, \int_{P_{U}} \varphi_{\omega}(p) \mathrm{d} \mu_{\omega}=1$,

$\omega(u)=\int_{-\infty}^{\infty} \lambda \mathrm{d} \omega\left(p_{\lambda}^{u}\right)=\int_{-\infty}^{\infty} \lambda\left(\int_{\bar{p}_{\lambda}^{u}} \varphi_{\omega}(p)\right) \mu_{\omega} \mathrm{d} \lambda$, and the function $\varphi_{\omega}: P_{U} \rightarrow R$, $\varphi_{\omega}\left(\omega_{p}\right)=\omega(p) \geq 0$ uniquely determines the state $\omega \in E_{U}$.

Let $u_{T}$ time observable and $\omega_{t}, t \in\left(\min \sigma_{u_{T}}, \max \sigma_{u_{T}}\right), \omega\left(u_{T}\right)=t$ states do not differ from each other on such observable values of which are invariant for a given particle in any state and determine its. Let $\varphi(p, t)=\varphi_{\omega_{t}}(p)$ then $\omega_{t}=\int_{P_{U}} p \varphi(p, t) \mathrm{d} \mu_{\omega}=\int_{P_{U}} p \varphi_{\omega}(p) \mathrm{d} \mu_{\omega}, \int_{P_{U}} \varphi(p, t) \mathrm{d} \mu_{\omega}=\int_{P_{U}} \varphi_{\omega}(p) \mathrm{d} \mu_{\omega}=1$. Let now $\phi(p, t)$ such function that $\phi(p, t)^{2}=\varphi(p, t) . \quad 0 \leq \varphi_{\omega}(p) \leq 1$, $\int_{P_{U}} \varphi_{\omega}(p) \mathrm{d} \mu_{\omega}=1$ it follows $\varphi_{\omega}(p)=\cos ^{2} \alpha(p), \phi(p, t)^{2}=\cos ^{2} \alpha(p, t)$ for all $t$ and $\phi(p, t)=\cos \alpha(p, t)$. Let's call the function $\phi(p, t)=\cos \alpha(p, t)$ amplitude of probability or wave function. This function uniquely determines the state $\omega_{t} \in E_{U}$.

During the experiment, a quantum system of elementary particles we observe in the time interval $t \in(\alpha, \beta) \subset\left(\min \sigma_{u_{T}}, \max \sigma_{u_{T}}\right)$ so elementary particle for us is continual sequence $\omega_{t}, t \in(\alpha, \beta) \subset\left(\min \sigma_{u_{T}}, \max \sigma_{u_{T}}\right)$. States $\omega_{t}$ not differ on observables which determine given particle. If we observe a particle in stationary motion, then

$$
\frac{\partial \phi(p, t)}{\partial t}=\frac{\partial \alpha(p, t)}{\partial t}=\text { const }
$$

It follows $\phi(p, t)=\cos (\alpha(p) t+\beta(p))$. We will be called $\alpha(p)$ and the $k(p)=-\frac{\beta(p)}{\left\|p-p_{0}\right\|}$ frequency and the wave number, respectively. Such 
$\phi(p, t)=\cos \left(\alpha(p) t-k(p)\left\|p-p_{0}\right\|\right)$ and we have the equation of wave with amplitude 1 .

Let wave functions: $\phi\left(p, p_{1}, t\right)=\cos \left(\alpha(p) t-k(p)\left\|p-p_{1}\right\|\right)$ and $\phi\left(p, p_{2}, t\right)=\cos \left(\alpha(p) t-k(p)\left\|p-p_{2}\right\|\right)$ define states of identical elementary particles.

Let identical elementary particles are such that their wave function interferes at the same phases:

$$
\begin{aligned}
& \phi\left(p, p_{1}, t\right)+\phi\left(p, p_{2}, t\right) \\
& =\cos \left(\alpha(p) t-k(p)\left\|p-p_{1}\right\|\right)+\cos \left(\alpha(p) t-k(p)\left\|p-p_{2}\right\|\right) \\
& =2 \cos \frac{\alpha(p) t-k(p)\left\|p-p_{1}\right\|+\alpha(p) t-k(p)\left\|p-p_{2}\right\|}{2} \\
& =\cos \frac{\alpha(p) t-k(p)\left\|p-p_{1}\right\|-\alpha(p) t+k(p)\left\|p-p_{2}\right\|}{2} \\
& =2 \cos \left(\alpha(p) t-\frac{k(p)\left(\left\|p-p_{1}\right\|+\left\|p-p_{2}\right\|\right)}{2}\right) \cos \frac{k(p)\left(\left\|p-p_{2}\right\|-\left\|p-p_{1}\right\|\right)}{2} \\
& \lim _{p_{1} \rightarrow p_{2}} \phi\left(p, p_{1}, t\right)+\phi\left(p, p_{2}, t\right) \neq 0 .
\end{aligned}
$$

This means that identical elementary particles, which interference occurs at the same phase can be in the same state. We well tell that the particles of such type obey the Bose-Einstein statistics.

Let identical elementary particles are such that their wave function interferes at the anti phase, we well have:

$$
\begin{aligned}
\phi\left(p, p_{1}, t\right)-\phi\left(p, p_{2}, t\right) \\
=\cos \left(\alpha(p) t-k(p)\left\|p-p_{1}\right\|\right)-\cos \left(\alpha(p) t-k(p)\left\|p-p_{2}\right\|\right) \\
=-2 \sin \frac{\alpha(p) t-k(p)\left\|p-p_{1}\right\|+\alpha(p) t-k(p)\left\|p-p_{2}\right\|}{2} \\
\cdot \sin \frac{\alpha(p) t-k(p)\left\|p-p_{1}\right\|-\alpha(p) t+k(p)\left\|p-p_{2}\right\|}{2} \\
=2 \sin \left(\alpha(p) t-\frac{k(p)\left(\left\|p-p_{1}\right\|+\left\|p-p_{2}\right\|\right)}{2}\right) \sin \frac{k(p)\left(\left\|p-p_{2}\right\|-\left\|p-p_{1}\right\|\right)}{2} \\
\lim _{p_{1} \rightarrow p_{2}} \phi\left(p, p_{1}, t\right)-\phi\left(p, p_{2}, t\right)=0 .
\end{aligned}
$$

This means that if a particles are in the same state, in this case the amplitude of probability, i.e. the squared modulus of the interfered wave function, is zero, this means that identical particles which interference occurs at the same phase cannot be in the same state.

For such particles we tell that they obey the Fermi-Dirac statistics, and particles of the second type obey the Fermi-Dirac statistics.

He maximum of the module of amplitude in interference of probability for Bose-Einstein particles will be reached when

$$
\left|\cos \frac{k(p)\left(\left\|p-p_{2}\right\|-\left\|p-p_{1}\right\|\right)}{2}\right|=1 .
$$


From it

$$
\frac{k(p)\left(\left\|p-p_{1}\right\|-\left\|p-p_{2}\right\|\right)}{2}=\pi n, n \in Z .
$$

From known formula $k(p)=\frac{2 \pi}{\Lambda(p)}$, we have:

$$
\frac{\left\|p-p_{1}\right\|-\left\|p-p_{2}\right\|}{\lambda(p)}=n, n \in Z .
$$

The fraction numerator there is the so-called stroke difference and the denominator is the wavelength.

Also, the maximum of the module of amplitude in interference of probability for Fermi-Dirac particles will be reached when

$$
\sin \left|\frac{k(p)\left(\left\|p-p_{2}\right\|-\left\|p-p_{1}\right\|\right)}{2}\right|=1 .
$$

From this

$$
\frac{k(p)\left(\left\|p-p_{1}\right\|-\left\|p-p_{2}\right\|\right)}{2}=\frac{\pi}{2}+\pi n, n \in Z .
$$

Such we will have:

$$
\frac{\left\|p-p_{1}\right\|-\left\|p-p_{2}\right\|}{\lambda(p)}=\frac{1}{2}+n, n \in Z .
$$

If $\phi\left(p, p_{1}, p_{2}, t\right)=\phi\left(p, p_{1}, t\right)+\phi\left(p, p_{2}, t\right)$, then $\phi\left(p, p_{1}, p_{2}, t\right)=\phi\left(p, p_{2}, p_{1}, t\right)$.

If $\phi\left(p, p_{1}, p_{2}, t\right)=\phi\left(p, p_{1}, t\right)-\phi\left(p, p_{2}, t\right)$, then $\phi\left(p, p_{1}, p_{2}, t\right)=-\phi\left(p, p_{2}, p_{1}, t\right)$.

\section{Operators of Creation End Annihilation of Quantum Physical System}

Let given the quantum system $\left(U, P_{\mathfrak{J}}, \mathfrak{I}\right)$, where $P_{\mathfrak{J}} \subset P_{U}$ Consider Tikhonov product $\Sigma=\otimes_{u \in \mathfrak{R}} \sigma_{u}$ on $\otimes_{u \in \mathfrak{R}} \sigma_{u}$ the we have prodact measure $\otimes_{u \in \mathfrak{R}} l_{u}$ [6] where $l_{u}$ Lebesgue measure on $\sigma_{u}$. We assume that the subsets $P_{\mathfrak{J}}, P_{U}$ measurable in $\underset{u \in \Re}{\otimes} \sigma_{u}$ with product measure $\otimes_{u \in \Re} l_{u}$.

Let $\{p\} \subset U$ be the set of one-dimensional projectors (one-dimensional projectors in operator representation of the $\sigma$-algebra $U$ ). This set of projectors we identified to the set of pure states $P_{U}$. Thus $\{p\} \approx P_{U}$. For every state $\omega \in \mathfrak{I}$ exist the function

$$
\varphi_{\omega}: P_{\mathfrak{J}} \rightarrow R, \varphi_{\omega}(p)=\omega(p) \geq 0 .
$$

Every such function uniquely defines a state on the $C^{*}$-algebra $U$.

Consider the Hilbert space $L_{2}\left(P_{\mathfrak{I}}\right)$ every function from this space defines a functional on the $C^{\star}$-algebra $U$, since $f \in L_{2}\left(P_{U}\right), f^{2} \geq 0$, and if we make the normalization, state on $C^{*}$-algebra $U$. We assume that the function $\varphi_{\omega}$ is in- 
tegrable, so $\varphi_{\omega} \in L_{2}\left(P_{U}\right)$ for all $\omega \in E_{U}$.

For tensor products $\underbrace{U \otimes U \otimes \cdots \otimes U}_{n}$ and $\underbrace{U^{*} \otimes U^{*} \otimes \cdots \otimes U^{*}}_{n}$ it's known that $\underbrace{U^{*} \otimes U^{*} \otimes \cdots \otimes U^{*}}_{n} \subset(\underbrace{U \otimes U \otimes \cdots \otimes U}_{n})^{*}$. If

$\omega_{1} \otimes \omega_{2} \otimes \cdots \otimes \omega_{n} \in \underbrace{U^{*} \otimes U^{*} \otimes \cdots \otimes U^{*}}_{n}$ and $\omega_{i} \in \mathfrak{I}, i=1,2, \cdots, n$ is states of the quantum system $\left(U, P_{\mathfrak{I}}, \mathfrak{I}\right)$, then $\omega_{1} \otimes \omega_{2} \otimes \cdots \otimes \omega_{n}$ represents the $n$ interacting particles of the system $\left(U, P_{\mathfrak{I}}, \mathfrak{I}\right)$ which are the in states $\omega_{1}, \omega_{2}, \cdots, \omega_{n}$. If $\omega_{1}=p_{1}, \omega_{2}=p_{2}, \cdots, \omega_{n}=p_{n}$ are the pure states, then

$$
\left\{p_{1} \otimes p_{2} \otimes \cdots \otimes p_{n}\right\} \approx\left\{\left(p_{1}, p_{2}, \cdots, p_{n}\right)\right\}=\underbrace{P_{\Im} \times P_{\mathfrak{J}} \times \cdots \times P_{\mathfrak{J}}}_{n} .
$$

Farther:

$$
\begin{aligned}
& \left\{p_{1} \otimes p_{2} \otimes \cdots \otimes p_{n}\right\} \subset(\underbrace{U \otimes U \otimes \cdots \otimes U}_{n})^{*}, \\
& \left\{p_{1} \otimes p_{2} \otimes \cdots \otimes p_{n}\right\} \approx\left\{\left(p_{1}, p_{2}, \cdots, p_{n}\right)\right\}=\underbrace{P_{\Im} \times P_{\mathfrak{I}} \times \cdots \times P_{\mathfrak{\Im}}}_{n} \subset(\underbrace{U \otimes U \otimes \cdots \otimes U}_{n})^{*} .
\end{aligned}
$$

Let $P_{(\underbrace{U \otimes U \otimes \cdots U}_{n})^{*}} \subset(\underbrace{U \otimes U \otimes \cdots \otimes U}_{n})^{*}$ the set of pure states, it's clear, that $\left\{p_{1} \otimes p_{2} \otimes \cdots \otimes p_{n}\right\} \subset P_{(\underbrace{U \otimes U \otimes \cdots \otimes U}_{n})^{*}}$ i.e. $\underbrace{P_{\mathfrak{J}} \times P_{\mathfrak{J}} \times \cdots \times P_{\mathfrak{J}}}_{n} \subset P_{(\underbrace{U \otimes U \otimes \cdots}_{n})^{(}} \cdot *$

If $\hat{\omega} \in E_{(\underbrace{U \otimes U \otimes \cdots \cup U}_{n})^{*}}$ for it, as above exist function $\hat{\varphi}_{\hat{\omega}}: P_{(\underbrace{U \otimes U \otimes \cdots \otimes U}_{n})^{*}} \rightarrow R$, $\hat{\varphi}_{\hat{\omega}}(\hat{p})=\hat{\omega}(\hat{p})$, where $\hat{p} \in P_{(\underbrace{U \otimes U \otimes \otimes U}_{n})^{*}}, \hat{\varphi}_{\hat{\omega}}(\hat{p}) \geq 0$, which uniquely defines state on $(\underbrace{U \otimes U \otimes \cdots \otimes U}_{n})^{*}$.

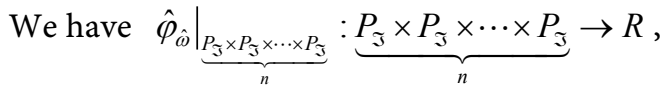
$\hat{\varphi}_{\hat{\omega}} \underbrace{}_{P_{P_{\mathfrak{S}} \times P_{\mathfrak{S}} \times \cdots \times P_{\mathfrak{S}}}} \in L_{2}(\underbrace{P_{\mathfrak{J}} \times P_{\mathfrak{\Im}} \times \cdots \times P_{\mathfrak{\Im}}}_{n})$, this Hilbert space is defined since on $\underbrace{P_{\mathfrak{J}} \times P_{\mathfrak{I}} \times \cdots \times P_{\mathfrak{\Im}}}_{n}$ we have measure $\underbrace{\left(\underset{u \in \Re}{\otimes} l_{u}\right) \otimes\left(\underset{u \in \Re}{\otimes} l_{u}\right) \otimes \cdots \otimes\left(\underset{u \in \Re}{\otimes} l_{u}\right)}_{n}$.

Every function $f^{2}$ from the space $L_{2}(\underbrace{P_{\Im} \times P_{\mathfrak{I}} \times \cdots \times P_{\mathfrak{\Im}}}_{n})$ defines a functional on the $C^{*}$-algebra $\underbrace{U \otimes U \otimes \cdots \otimes U}$, since, $f^{2} \geq 0$, and if we make the normalization-state on $C$-algebra

$$
\underbrace{U \otimes U \otimes \cdots \otimes U}_{n} .
$$


Consider co-chain complex

$\left(C^{n}(\underbrace{P_{\mathfrak{I}} \times P_{\mathfrak{I}} \times \cdots \times P_{\mathfrak{S}}}_{n}, R), d^{n}\right)=\left(L_{2}(\underbrace{P_{\mathfrak{I}} \times P_{\mathfrak{I}} \times \cdots \times P_{\mathfrak{I}}}_{n}), d^{n}\right)$ where the boundary operator $d^{n}: C^{n}(\underbrace{P_{\Im} \times P_{\Im} \times \cdots \times P_{\Im}}_{n}, R) \rightarrow C^{n+1}(\underbrace{P_{\Im} \times P_{\Im} \times \cdots \times P_{\Im}}_{n}, R)$ defined by formula $d^{n}\left(f\left(p_{1}, p_{2}, \cdots, p_{n}\right)\right)=\sum_{i=1}^{n+1}(-1)^{i} f\left(p_{1}, p_{2}, \cdots, \hat{p}_{i}, \cdots, p_{n+1}\right)$ [7].

We have also the chain complex

$C_{n}(\underbrace{P_{\Im} \times P_{\mathfrak{J}} \times \cdots \times P_{\mathfrak{\Im}}}_{n}, R)=L_{2}(\underbrace{P_{\Im} \times P_{\mathfrak{\Im}} \times \cdots \times P_{\mathfrak{\Im}}}_{n})^{*}$ where the boundary operator $d_{n}: C_{n}(\underbrace{P_{\mathfrak{I}} \times P_{\mathfrak{I}} \times \cdots \times P_{\mathfrak{J}}}_{n}, R) \rightarrow C_{n-1}(\underbrace{P_{\mathfrak{I}} \times P_{\mathfrak{I}} \times \cdots \times P_{\mathfrak{I}}}_{n}, R)$ defined by

formula $d_{n}\left(f^{*}\right)=f^{*} \circ d^{n-1}$, where $f^{*} \in L_{2}(\underbrace{P_{\Im} \times P_{\mathfrak{I}} \times \cdots \times P_{\mathfrak{3}}}_{n})^{*} \approx L_{2}(\underbrace{P_{\mathfrak{I}} \times P_{\mathfrak{I}} \times \cdots \times P_{\mathfrak{3}}}_{n})$.

For this co-chain and chain complexes we have:

$C^{n}(\underbrace{P_{\mathfrak{I}} \times P_{\mathfrak{I}} \times \cdots \times P_{\mathfrak{J}}}_{n}, R)=C_{n}(\underbrace{P_{\mathfrak{I}} \times P_{\mathfrak{I}} \times \cdots \times P_{\mathfrak{J}}}_{n}, R) \approx L_{2}(\underbrace{P_{\mathfrak{I}} \times P_{\mathfrak{I}} \times \cdots \times P_{\mathfrak{J}}}_{n})$.

Each function $f$ from the space $L_{2}(\underbrace{P_{\Im} \times P_{\Im} \times \cdots \times P_{\mathfrak{\Im}}}_{n})$ defines a functional on the $C^{*}$-algebra $\underbrace{U \otimes U \otimes \cdots \otimes U}$, including a functionals of this kind $\omega_{1} \otimes \omega_{2} \otimes \cdots \otimes \omega_{n}$ which represents the system of $n$ interacting particles of the system $\left(U, P_{\mathfrak{I}}, \mathfrak{I}\right)$.

The operator $d^{n}: C^{n}(\underbrace{P_{\mathfrak{I}} \times P_{\mathfrak{I}} \times \cdots \times P_{\mathfrak{J}}}_{n}, R) \rightarrow C^{n+1}(\underbrace{P_{\mathfrak{I}} \times P_{\mathfrak{I}} \times \cdots \times P_{\mathfrak{J}}}_{n}, R)$

translates function which represent the system of $n$ interacting particles $\omega_{1} \otimes \omega_{2} \otimes \cdots \otimes \omega_{n}$ to function which represent the system of $n+1$ interacting particles $\omega_{1} \otimes \omega_{2} \otimes \cdots \otimes \omega_{n+1}$.

Conversely the operator

$d_{n}: C_{n}(\underbrace{P_{\Im} \times P_{\mathfrak{I}} \times \cdots \times P_{\mathfrak{\Im}}}_{n}, R) \rightarrow C_{n-1}(\underbrace{P_{\Im} \times P_{\mathfrak{I}} \times \cdots \times P_{\mathfrak{\Im}}}_{n}, R)$ translates function which represent the system of $n$ interacting particle $\omega_{1} \otimes \omega_{2} \otimes \cdots \otimes \omega_{n}$ to function which represent the system of $n-1$ interacting particles $\omega_{1} \otimes \omega_{2} \otimes \cdots \otimes \omega_{n-1}$.

From what has been said, it follows that the operators $d^{n}$ and $d_{n}$ are operators of creation and annihilation, respectively.

The co-chain complex 


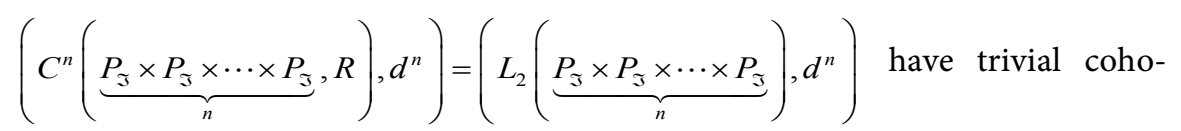
mologies [7].

Local trivial function $f$ on $\underbrace{P_{\Im} \times P_{\Im} \times \cdots \times P_{\Im}}_{n}$ is such function for which exist open cover $\left\{O_{\alpha}\right\}$ of $P_{U}$ that $f$ is zero on any $\left(p_{1}, p_{2}, \cdots, p_{n}\right) \in \underbrace{P_{\Im} \times P_{\mathfrak{I}} \times \cdots \times P_{\mathfrak{J}}}_{n}$, where $p_{i} \in O_{\alpha}, i=1,2, \cdots, n$ and $O_{\alpha}$ some element of cover $\left\{O_{\alpha}\right\}$.

The closure of subset local trivial functions in $C^{n}(\underbrace{P_{\Im} \times P_{\Im} \times \cdots \times P_{\Im}}_{n}, R)=L_{2}(\underbrace{P_{\Im} \times P_{\Im} \times \cdots \times P_{\Im}}_{n})$ form sub Hilbert space, denote it by $C_{0}^{n}(\underbrace{P_{\Im} \times P_{\widetilde{I}} \times \cdots \times P_{\Im}}_{n}, R)$. It's clear, that

$$
d^{n}\left(C_{0}^{n}(\underbrace{P_{\mathfrak{J}} \times P_{\mathfrak{I}} \times \cdots \times P_{\mathfrak{\Im}}}_{n}, R)\right) \subset C_{0}^{n+1}(\underbrace{P_{\mathfrak{\Im}} \times P_{\mathfrak{J}} \times \cdots \times P_{\mathfrak{3}}}_{n}, R) .
$$

If
$\bar{C}^{n}(\underbrace{P_{\mathfrak{I}} \times P_{\mathfrak{I}} \times \cdots \times P_{\mathfrak{I}}}_{n}, R)=C^{n}(\underbrace{P_{\mathfrak{I}} \times P_{\mathfrak{I}} \times \cdots \times P_{\mathfrak{I}}}_{n}, R) / C_{0}^{n}(\underbrace{P_{\mathfrak{I}} \times P_{\mathfrak{I}} \times \cdots \times P_{\mathfrak{I}}}_{n}, R)$ then we have co chain complex $\left(\bar{C}^{n}(\underbrace{P_{U} \times P_{U} \times \cdots \times P_{U}}_{n}, R), \bar{d}^{n}\right)$ with non trivial cohomologies [7].

If we consider annihilation operators for only local interacting particle system, then Chain complex $\left(\bar{C}_{n}(\underbrace{P_{\mathfrak{J}} \times P_{\mathfrak{\Im}} \times \cdots \times P_{\mathfrak{\Im}}}_{n}, R)^{*}, \bar{d}_{n}\right)$ where $\bar{d}_{n}$ defined by formula $\bar{d}_{n}\left(f^{*}\right)=f^{*} \circ \bar{d}^{n}, f^{*} \in \bar{C}_{n}(\underbrace{P_{\Im} \times P_{\mathfrak{I}} \times \cdots \times P_{\mathfrak{I}}}_{n}, R)^{*}$ defines homological structure of space $p_{\mathfrak{I}}[7]$.

From all that has been said follows: In quantum physical system $\left(U, P_{\mathfrak{I}}, \mathfrak{I}\right)$ operators creation and annihilation defines co-homological and homological structures physical space $P_{\mathfrak{J}}$, respectively.

\section{Results}

In this article was obtained following results:

1) Built for the wave function of a quantum system $\left(U, P_{\mathfrak{I}}, \mathfrak{I}\right)$.

2) From the constructed wave function, the separation of elementary particles into the Bose-Einstein and Fermi-Dirac classes was made.

3) Operators of creation and annihilation of elementary particles are presented as co-boundary and boundary operators of co-chain and chain complexes over space $P_{\mathfrak{I}}$, respectively. 


\section{Conflicts of Interest}

The author declares no conflicts of interest regarding the publication of this paper.

\section{References}

[1] Bogolyubov, H.H., Logunov, A.A., Oksak, A.I. and Todorov, I.T. (1987) General Principles of the Quantum Field Theories. Nauka, Moscow. (In Russian)

[2] Bratteli, O. and Robinson, D. (1982) Operator Algebras and Quantum Statistical Mechanics. Nauka, Moscow. (In Russian)

[3] Araki, H. (1999) Mathematical Theory of Quantum Fields. Oxford Science Publication, Oxford.

[4] Mumladze, M. and Zerakidze, Z. (2015) The Criteria of Checking Hypotheses of Quantum States of Quantum Physical System. Journal of Algebra, Number Theory: Advances and Applications, 14, 57-65.

[5] Liusterniik, L.A. and Sobolev, V.I. (1965) Elements of Functional Analyses. Nauka, Moscow. (In Russian)

[6] Bell, J. (2015) Infinite Product Measures. Departments of Mathematics, University of Toronto, Toronto.

[7] Edwin, H. (1966) Spanier Algebraic Topology. McGraw-Hill Book Company, New York. 